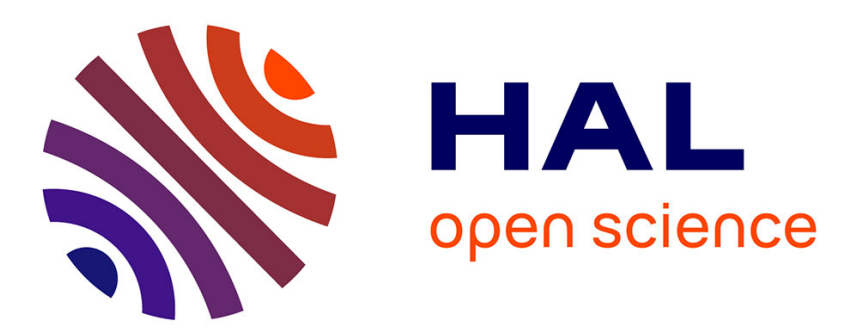

\title{
On Continuation Methods for Non-Linear Bi-Objective Optimization: Certified Interval-Based Approach
}

Benjamin Martin, Alexandre Goldsztejn, Laurent Granvilliers, Christophe Jermann

\section{- To cite this version:}

Benjamin Martin, Alexandre Goldsztejn, Laurent Granvilliers, Christophe Jermann. On Continuation Methods for Non-Linear Bi-Objective Optimization: Certified Interval-Based Approach. 2013. hal00878729

\section{HAL Id: hal-00878729 \\ https://hal.science/hal-00878729}

Preprint submitted on 30 Oct 2013

HAL is a multi-disciplinary open access archive for the deposit and dissemination of scientific research documents, whether they are published or not. The documents may come from teaching and research institutions in France or abroad, or from public or private research centers.
L'archive ouverte pluridisciplinaire HAL, est destinée au dépôt et à la diffusion de documents scientifiques de niveau recherche, publiés ou non, émanant des établissements d'enseignement et de recherche français ou étrangers, des laboratoires publics ou privés. 


\title{
On Continuation Methods for Non-Linear Bi-Objective Optimization: Certified Interval-Based Approach
}

\author{
Benjamin Martin • Alexandre Goldsztejn • \\ Laurent Granvilliers · Christophe Jermann
}

the date of receipt and acceptance should be inserted later

\begin{abstract}
The global optimization of constrained Non-Linear Bi-Objective Optimization problems (NLBOO) aims at covering their Pareto-optimal front which is in general a manifold in $\mathbb{R}^{2}$. Continuation methods can help in this context as they can follow a continuous component of this front once an initial point on it is provided. They constitute somehow a generalization of the classical scalarizing framework which transforms the bi-objective problem into a parametric monoobjective problem. Recent works have shown that they can play a key role in global algorithms dedicated to bi-objective problems, e.g. population based algorithms, where they allow discovering large portions of locally Pareto optimal vectors, which turns out to strongly support diversification.

In this paper, we provide a survey on continuation techniques in global optimization methods for NLBOO, which allow discovering large portions of locally Pareto-optimal solutions. We also propose a rigorous active set management strategy on top of a previously proposed certified continuation method based on interval analysis, and illustrate it on a challenging bi-objective problem.
\end{abstract}

Keywords Non-Linear Bi-Objective Optimization · Continuation · Interval Analysis · Constraints Activity

\section{Introduction}

Non-Linear (Constrained) Bi-Objective Optimization (NLBOO) is the problem of simultaneously optimizing two criteria and can be formally defined as:

$$
\left[\begin{array}{ll}
\min & f(x) \\
\text { s.t } & g(x) \leq 0 \text { and } h(x)=0 \\
& x \in \mathbb{R}^{n}
\end{array}\right]
$$

Benjamin Martin, Laurent Granvilliers, Christophe Jermann

Université de Nantes, LINA (UMR 6241), France, E-mail: name.surname@univ-nantes.fr,

Alexandre Goldsztejn

CNRS, LINA (UMR 6241), France, E-mail: Alexandre.Goldsztejn@gmail.com 
with $x \in \mathbb{R}^{n}$ the decision variables, $f: \mathbb{R}^{n} \rightarrow \mathbb{R}^{2}$ the two objective functions, $g: \mathbb{R}^{n} \rightarrow \mathbb{R}^{p}$ inequality constraints and $h: \mathbb{R}^{n} \rightarrow \mathbb{R}^{q}$ equality constraints. Functions $f, g$ and $h$ can be non-linear.

The feasible region $\mathcal{X}$ is the set of decision vectors that satisfy all the constraints, i.e., $\mathcal{X}:=\left\{x \in \mathbb{R}^{n} \mid g(x) \leq 0\right.$ and $\left.h(x)=0\right\}$. Its image $\mathcal{F}=f(\mathcal{X})$ in the objective space is called the feasible objective region. A decision vector $x \in \mathcal{X}$ dominates another decision vector $y$ if $f(x) \leq f(y)$ (componentwise) and $f(x) \neq f(y)$. Then, $x$ is called Pareto-optimal if it feasible and not dominated by some other feasible decision vector, the set of these vectors being denoted by $\mathcal{X}^{*}$. Intuitively, they correspond to optimal trade-offs between the two objectives. They include the minimizers $x_{i}^{*}$ of each individual objective $f_{i}$, whose value $f_{i}^{*}=f_{i}\left(x_{i}^{*}\right)$ cannot be dominated. The utopia point $f^{*}=\left(f_{1}\left(x_{1}^{*}\right), f_{2}\left(x_{2}^{*}\right)\right)$ is infeasible in general, inducing the necessity of a trade-off. As in mono-objective optimization, local Pareto-optimal vectors can be non globally Pareto-optimal. The global optimization of Problem (1) aims at finding globally Pareto optimal vectors $\mathcal{X}^{*}$. Local and global Pareto-optimal vectors generally form manifolds of dimension 1 in the case of (continuous) nonlinear bi-objective optimization. This is a major issue for global algorithms, whose diversification aims at both avoiding local optimizers and spreading along these manifolds. In this context, continuation methods can turn out to be of critical importance in order to locally compute those manifolds.

Continuation methods [1] explore step by step a manifold, usually defined as the solution set of an under-constrained system of equations $F(x)=0$ with $F: \mathbb{R}^{a+b} \rightarrow \mathbb{R}^{a}$, and $b=1$ for one-parameter continuation, which is the case in the present paper. It has a wide range of applications, e.g. polynomial root finding via homotopy [2], nonlinear eigenvalue problems [3], parametric optimization [33], robot path planning [23,31], etc. And, multi-objective optimization (e.g., [15]). The main limitation for their application to multi-objective optimization is that they generally cannot handle constrained problems. Indeed the presence of inequality constraints induces non-differentiable break points (singularities) in the followed manifold. Passing those singularities requires both their detection and handling correctly the constraints activity.

Several certified continuation methods have been proposed: Some concentrate on deriving a guaranteed step size, like [2] based on Smale's $\alpha$-theory [42], [8] based on interval analysis or [4] based on the reach of a manifold. Others perform rigorous computations using interval analysis and employ solution existence procedures to certify the enclosure of connected portions of the followed manifold [16, 22 ]. Although more expensive than non certified methods, they can be used within a global optimization algorithm, since the price of rigorousness can be small with respect to the price of globality, while certification is a real advantage, e.g. for enforcing bounds. Continuation alone, even rigorous, is however not sufficient for the global optimization of Problem (1) since the Pareto front may consist of several disjoint components. It must be coupled with a global optimizer whose role is to attain each component, the continuation role being to cover the attained components. Hence each assume a different aspect of the diversification process.

In this paper, we first provide a survey of continuation methods used for multiobjective optimization, providing an homogeneous view over several scalarizingbased techniques (Section 2), then explaining why continuation based on first-order conditions is preferable and how it has been used until now. We conclude there is a lack in handling properly inequality constraints and we propose an adaptation 
of the rigorous continuation method in [22] to this end (Section 3), illustrating a possible usage for the global optimization of Problem (1).

\section{Survey of Continuation Approaches for Bi-Objective Optimization}

A Continuation Viewpoint on Scalarizing Methods. A common and traditional approach when tackling NLBOO is to transform the problem (1) into a monoobjective problem, parameterized such that its optimizers compose a subset of $\mathcal{X}^{*}$. This process is called scalarization. Scalarizing methods generally apply to problems with more than two objectives, though for the sake of clarity we will present them in this context, without loss of generality.

Well-known scalarizing methods are the Weighted-Sum (WS) and the $\varepsilon$-constraint $(\varepsilon \mathrm{C})$, see $[27,6]$. They induce the following problems:

$$
W S \equiv\left[\begin{array}{ll}
\min & \lambda_{1} f_{1}(x)+\lambda_{2} f_{2}(x) \\
\text { s.t } & x \in \mathcal{X}
\end{array}\right] \text { and } \varepsilon C \equiv\left[\begin{array}{ll}
\min & f_{2}(x) \\
\text { s.t } & f_{1}(x) \leq \varepsilon \\
& x \in \mathcal{X}
\end{array}\right]
$$

where $\lambda_{1}+\lambda_{2}=1$. These problems induce new constraints and parameters that control the targeted trade-off in $\mathcal{F}^{*}$. Capturing a specific trade-off using particular parameters values is not easy, and sometimes impossible. E.g., the weighted sum can not compute solutions whose objective vector is not on the convex hull of $\mathcal{F}$. In order to deal with such limitations, more complex methods have been defined.

The Normal Boundary Intersection (NBI) from Das [5] relies upon the Convex Hull of Individual Minima (CHIM), i.e. the line in the objective space containing the minima $f\left(x_{1}^{*}\right)$ and $f\left(x_{2}^{*}\right)$. The scalarized problem by NBI can then be stated as follows:

$$
N B I \equiv\left[\begin{array}{ll}
\max & t \\
\text { s.t } & f_{\lambda}+t u=f(x) \\
& x \in \mathcal{X}
\end{array}\right],
$$

with $f_{\lambda}=\lambda_{1} f\left(x_{1}^{*}\right)+\lambda_{2} f\left(x_{2}^{*}\right), \lambda_{1}+\lambda_{2}=1, u=\left(f^{*}-f\left(x_{1}^{*}\right)\right)+\left(f^{*}-f\left(x_{2}^{*}\right)\right)$ and $t$ an additional variable. The point $f_{\lambda}$ belongs to the CHIM and the vector $u$ is a quasi normal vector of the CHIM directing towards the utopia point $f^{*}$. Therefore, the NBI consists of finding the objective vector in $\mathcal{F}$ the farthest from $f_{\lambda}$ along the direction $u$. The NBI is more reliable than WS or $\varepsilon \mathrm{C}$, but it may produce non Pareto-Optimal solutions [5].

The (Normalized) Normal Constraint (NC) from Messac et al. [24,26] combines the use of the CHIM and the principle of the $\varepsilon \mathrm{C}$ method. The NC scalarization of (1) is defined as follows:

$$
N C \equiv\left[\begin{array}{ll}
\min & f_{2}(x) \\
\text { s.t } & \left(f\left(x_{2}^{*}\right)-f\left(x_{1}^{*}\right)\right)^{T}\left(f(x)-f_{\lambda}\right) \leq 0 \\
& x \in \mathcal{X}
\end{array}\right]
$$

As in $\varepsilon \mathrm{C}$, one objective is minimized while the other is put in a constraint defining a cutting plane in the objective space, normal to the CHIM. Contrarily to NBI, NC induces only one additional inequality constraint, although the two methods are closely related. Insights on how to advantageously select one of the two methods is given in [18]. Both NBI and NC have limitations, for instance when considering 
more than two objectives. Some improvements of these two techniques have been proposed $[38,28]$.

Other scalarizing techniques have been proposed, like Physical Programming (PP) which introduces decision maker preferences in the process, see [25, 43]; or the Directed Search Domain, see [7], in which additional parameters are introduced in order to orient search domains defined in an NC and PP fashion.

In the context of global optimization, all these methods have in common that they require solving several instances of the scalarized problem for different parameter values. This problem is clearly related to Parametric optimization where a parameter-dependent mono-objective optimization problem is solved for different parameter values, the goal being to observe how the optimum vary with respect to the parameters. It is usualy supposed that a slight change of the parameters values leads to a slight change of the optimum. Hence, starting from one (globally) computed solution, a basic, but efficient, iterative process is to shift the parameters values and to apply a local solver on the induced new problem, starting from the previously computed optimum [27]. This iterative process can be seen as a form of continuation whose attempt is to locally approximate the manifold of Paretooptimal solutions. However, it has several drawbacks. First, being local in nature, the process can easily diverge from the global optimizer of NLBOO. It may be require to couple the technique with a global approach, e.g. as in [36]. Second, its performances strongly depend on the selected scalarization and the employed local solver. Finally, it is not obvious how to sample the parameters values so as to achieve a well distributed sample of Pareto-optimal solutions.

Another approach in Parametric optimization is based on the use of continuation on the system of equations defined by the Karush-Kuhn-Tucker or the Fritz-John first-order conditions [35,33]. The single parameter case can indeed be assimilated to Bi-Objective optimization, as remarked in [34]. They propose a continuation method based on the WS scalarization of (1) and that implements a strategy for detecting active set change, a required feature to deal with inequality constraints since those changes are singular for the system of first-order conditions. Other singularities can still occur, such as the loss of constraints complementarity, see [21]. Moreover, this method uses second order otpimality conditions to track (local) minima of the WS scalarization. Hence, it can not track optimal solutions that are on the non-convex parts of the Pareto-optimal front.

Continuation Methods in Bi-Objective Optimization. From the work on continuation methods in parametric optimization, Hillermeier [15] has proposed a general scheme of continuation approaches for Non-Linear Multi-Objective optimization. Although it does not consider inequality constraints, any number of objectives can be used. The process consists of locally tracking the manifold of Pareto-optimal solutions, once a solution is found, implicitly defined by the system of KarushKuhn-Tucker or Fritz-John first order optimality conditions of (1). This system can be defined as follows:

$$
F(x, \lambda, r, s)=\left(\begin{array}{l}
\nabla f(x) \lambda+\nabla g(x) r+\nabla h(x) s \\
(\forall i=1, \ldots, p) g_{i}(x) r_{i} \\
(\forall i=1, \ldots, q) h_{i}(x) \\
\lambda^{T} \lambda+r^{T} r+s^{T} s-1
\end{array}\right)=0,
$$


with $\lambda \in \mathbb{R}_{+}^{2}, r \in \mathbb{R}_{+}^{p}$ and $s \in \mathbb{R}^{q}$. As previously said, the continuation process is a local technique that requires an initial Pareto optimal solution. Hence, it has to be coupled with multi-objective global solvers.

One of the first offspring of this scheme is the recovering algorithm of Schütze et al. [40]. Continuation is used as a repair operator inside a global sub-division method. This method sub-divides the search and objective spaces in boxes that are discarded if no solution is found within them. The discarding process is not guaranteed: a box can be removed while it contains Pareto-optimal solutions. The recovering algorithm helps to recover those missing boxes using solutions from neighboring boxes and a continuation process. A similar recovering algorithm is used by Shütze et al. inside a particle swarm optimizer [39]. The aim of the recovering algorithm is here to diversify and improve the spread of the population of the evolutionary metaheuristic. As for Hillermeier [15], this technique has not been applied to problems with inequality constraints.

Harada et al. in [13] have also proposed to combine a metaheuristic with a continuation method. This continuation process, called Pareto Path Following $(\mathrm{PPF})$, is a predictor-corrector algorithm. The predictor step constructs a new solution using the gradient of the objectives as an initial direction of continuation, which is repaired by the Pareto-descent repair operator [14] and a gradient projection method for handling constraints. The corrector step uses the Paretodescent local search [12]. The authors introduce two new performance measures for bi-objective optimization (but the many-objective case can be easily derived): inter-curve coverage which measures the ability of a method to reach each of the disconnected curves of Pareto-optimal solutions, and intra-curve coverage which measures the spread of solutions on these components. Eventually, a curved-based Genetic Algorithm is proposed for bi-objective problems. This algorithm considers (locally) Pareto optimal curves as atomic elements of the population. A curve is represented as a cluster of solutions. Genetic crossover operators are applied on solutions from different curves in order to find solutions on non-discovered curves. This method supposes that once a Pareto-optimal solution is found, then all the solutions belonging to the same curve of (locally) Pareto optimal solutions can be obtained by continuation. Hence, the genetic operators focus on inter-curve coverage whereas the PPF focuses on intra-curve coverage. This technique shows better performances at the end of the run than classic Genetic Algorithm, but it requires more evaluations of the objectives. Although the three objectives and the constrained case are discussed in [13], only one experiment on a bi-objective bound-constrained problem is shown.

As stated in [1], solving a system as (5) by continuation is equivalent to solving a specific system of differential equations. Pereyra [30] has proposed to transform bi-objective unconstrained convex problems into 2-point boundary value problems. In that case, the first-order optimality conditions can be defined as:

$$
(1-\lambda) \nabla f_{1}(x)+\lambda \nabla f_{2}(x)=0,
$$

with $\lambda \in[0,1]$. The problem consists of finding the parametric curve $x(\lambda):[0,1] \rightarrow$ $\mathbb{R}^{n}$ such that $(x(\lambda), \lambda)$ satisfies $(6)$ for all $\lambda$. Since only convex problems are considered, this curve represents the Pareto-optimal solutions. It is computed as the solution curve of the following two-points boundary-value problem:

$$
\dot{x}(\lambda)=-H_{x}^{-1}(x) H_{\lambda}(x), x(0)=x_{1}^{*}, x(1)=x_{2}^{*},
$$


where $H_{x}(x)=(1-\lambda) \nabla^{2} f_{1}(x)+\lambda \nabla^{2} f_{2}(x)$ and $H_{\lambda}(x)=-\nabla f_{1}(x)+\nabla f_{2}(x)$. As for the NBI scalarization, solving (7) requires to find the minimum of each objective $x_{1}^{*}$ and $x_{2}^{*}$. The proposed continuation process is a predictor-corrector. The prediction increments the parameter $\lambda$ by $\delta_{\lambda}$, and uses the previous corrected solutions as an initial guess. The correction step is the application of the Newton method on the system (6). This process produces a set of points that covers the Pareto-optimal curve. As the time step parameter is $\lambda$, the technique suffers the same drawback of the WS, hence the method is restricted to convex problems. In addition to this continuation method, Pereyra [30] has proposed additional constraints ensuring an homogeneous coverage, in the decision or objective space. A parallel algorithm inspired by the continuation process is also proposed, and shows similarity with using NBI scalarization. Finally, although the three objectives case is discussed, all these techniques are asserted only on bi-objective problems.

Another method based on solving a system of differential equation by continuation is the method of Potschka et al. [32]. This method is based on the NBI or NC scalarization of (1) in the bi-objective case. More precisely, the considered scalarized problem is as follows:

$$
\left[\begin{array}{ll}
\min & f_{2}(x) \\
\text { s.t } & g(x) \leq 0 \text { and } h(x)=0 \\
& u^{t}(f(x)-\hat{f}(v))=0
\end{array}\right]
$$

with $\hat{f}(v)=v f\left(x_{1}^{*}\right)+(1-v) f\left(x_{2}^{*}\right)$ and $u=f\left(x_{1}^{*}\right)-f\left(x_{2}^{*}\right)$. The parameter $v$ vary within $[0,1]$. As in [34], an active set of constraints $\mathcal{A}$ is used to handle the change of activity of inequalities. Therefore, active inequalities are considered as equalities, while inactive ones are not considered. Let $h_{e}(x, v)$ be the additional equality $u^{t}(f(x)-\hat{f}(v))$. Let $L\left(x, r_{\mathcal{A}}, s, s_{e}, v\right)=f_{2}(x)+r_{\mathcal{A}} g_{\mathcal{A}}(x)+s h(x)+s_{e} h_{e}(x, v)$ the Lagrangian of (8) considering the active set $\mathcal{A}$, with $g_{\mathcal{A}}$ and $r_{\mathcal{A}}$ being the vector of active constraints and multipliers. The induced first-order conditions are given by:

$$
F\left(x, r_{\mathcal{A}}, s, s_{e}, v\right)=\left(\begin{array}{l}
\nabla_{x} L\left(x, r_{\mathcal{A}}, s, s_{e}, v\right) \\
g_{\mathcal{A}}(x) \\
h(x) \\
h_{e}(x, v)
\end{array}\right)=0 .
$$

Denoting $y=\left(x, r_{\mathcal{A}}, s, s_{e}\right)$, Potschka et al. [32] have proposed to determine the curve of solutions of (9), parameterized by $v$, solving the following ordinary differential equation system:

$$
\dot{y}(v)=\left(\nabla_{y} F(y, v)\right)^{-1}\left(\nabla_{v} F(y, v)\right) .
$$

The system is solved by continuation through an integrator applied on the variable $v$. The set of active constraints is managed such that the process builds feasible and (locally) Pareto-optimal vectors. This management of $\mathcal{A}$ is implemented using the event detection of the integrator, which triggers changes of the active set on conditions similar to [34] (i.e. studying the change of the sign of a constraint or of its multiplier). When a change is detected at a solution $x$, the active set is updated according to the constraints that are active at $x$. As in [30], this continuation technique moves along a fixed parameter, here $v$. Hence, it can encounter turning points, and stops tracking the curve, when the front is perpendicular to the CHIM. 
One limitation of continuation methods is that they usually require to build the tangent space of the tracked manifold at each iterate. From a system of $n$ equations $F(x)=0$, the complexity of such an operation is $O\left(n^{3}\right)$. Although the complexity is polynomial, it tends to be very expensive to build tangent space of problems involving thousands of variables. Ringkamp et al. [37] have proposed a tangent space approximation technique in the context of continuation for multi-objective optimization. This technique reduces the complexity of the tangent computation to $O\left(n^{2}\right)$. This approximation method can be applied for any problems where continuation can be used.

Other continuation approaches to mulit-objective optimization not based on solving (5) also exist. All the continuation methods presented before requires the use of the gradient (or hessian) of objectives and constraints. In the case where gradients are not available, Schütze et al. [41] have proposed to use the descent method HCS from Lara et al. [17] in order to perform the continuation on unconstrained multi-objective problems. This technique is based on the observation that, without constraints, there is less chances in finding a direction that improves all objectives at a solution $x$ if this solution gets closer to a (locally) Pareto-optimal front. On the contrary, the chances of finding a direction of trade-off (i.e. that improves one objective and deteriorates the other) increases. The idea in [41] is to build a predictor-corrector technique which uses the HCS to build trade-off directions for predicting new solutions along the Pareto-optimal curve, and to build improving directions for correcting the predicates. The HCS used in [41] constructs these two kind of directions by using gradient approximation techniques. These approximations are also used to determine the length of the continuation step. Although this can, in theory, be used to solve unconstrained problems with any number of objectives, only the bi-objective case is asserted.

Finally, Lovison [19] has proposed a study of the global characterization of Pareto-optimal manifolds, by using first-order conditions different from the ones of Karush-John. This characterization consists of a piecewise linear approximation of these manifolds using a Delaunay tessellation of the search space, i.e. a decomposition of the search space in simplices. Piecewise linear continuation is used to determine the simplices cut by the manifold. In addition, a process shrinking simplices around the manifold is proposed. The treated problems are unconstrained, but the methods can be adapted to deal with equalities. Any number of objectives can be considered. The technique has some limitations. First, the complexity of the Delaunay tessellation is exponential with the number of variables, hence the process is limited in problem size. Second, the initial tessellation must be thin enough in order to guarantee each disconnected components of the Pareto-optimal manifold are cut by the at least one simplex. Some issues have been partially answered by Lovison [20]. The author indeed propose a simpler globally convergent version of the algorithm using a decomposition of the search space by regular (equilateral) triangles, dedicated to solve unconstrained problems with 2-variables and 2-objectives. The method shares some similarity with [40]: the former captures the manifold of Pareto-optimal solutions by means of regular triangles, the latter by means of hyper-rectangles.

Conclusion. All the techniques presented here show a promising direction towards the design of efficient local methods to use inside a global bi-objective optimization solver. On non-convex problems, methods that fix a priori a parameter to control 
the continuation, such as scalarizing approaches or the ODE-based continuation, share a common drawback: They are faced to turning points that stops the continuation. Techniques based on continuing the first order conditions (5) without using a fixed parameter, like Hillermeier [15], remove this drawback.

\section{Certified Continuation for Constrained Bi-Objective Optimization}

Using the certified continuation method [22] for performing the continuation on the first order conditions allows in particular certifying the feasibility, which can turn out to be critical e.g. when feasible vectors are to be used as bounds in a global algorithm. However, neither [22] nor Hillermeier [15] handle active constraints, leading to failure of the continuation process at active constraints change. The correct handling of such active constraint change allows discovering locally larger sets of locally optimal solutions, hence

In this section, we present an extension of the certified continuation algorithm ParCont defined in [22], to be used on bi-objective problems with correct handling of active constraints change, which is inspired by [34]. This method is based on interval analysis and parallelotope domains. A brief introduction to these notions is given. We refer to $[29,9]$ for more details.

\subsection{Preliminaries}

Interval Analysis and Parallelotope Domains. An interval is a closed connected subset of $\mathbb{R}$. Intervals are denoted by boldface symbols, e.g. $\boldsymbol{x} \subseteq \mathbb{R}$. Lower and upper bounds of an interval $\boldsymbol{x}$ are denoted by $\underline{x} \in \mathbb{R}$ and $\bar{x} \in \mathbb{R}$, with $\underline{x} \leq \bar{x}$, i.e. $\boldsymbol{x}=[\underline{x}, \bar{x}]=\{x \in \mathbb{R} \mid \underline{x} \leq x \leq \bar{x}\}$. A real number $x$ will be identified with the degenerated interval $[x, x]$. A box $\boldsymbol{x} \subseteq \mathbb{R}^{n}$ denotes a vector of intervals. Interval arithmetic extends elementary operations and functions to interval arguments following the containment principle: any possible real result must be enclosed in the returned interval result. An interval extension of any function can then be defined as its evaluation using interval arithmetic. Interval analysis can be used to solve systems of equations $F(x)=0$. For example, interval Newton methods are used to contract an initial interval or box around a solution of the system. Numeric proofs of existence and unicity of solutions are build through an interval Newton operator.

A parallelotope $\widehat{\boldsymbol{x}}$ is the image of a box $\boldsymbol{w}$ through an affine map $w \rightarrow C w+\tilde{x}$. It is defined by a triple $(C, \boldsymbol{w}, \tilde{x})$, where $C \in \mathbb{R}^{n \times n}, \boldsymbol{w} \subseteq \mathbb{R}^{n}$ and $\tilde{x} \in \mathbb{R}^{n}$, whose corresponding parallelotope is $\widehat{\boldsymbol{x}}=\left\{C w+\tilde{x} \in \mathbb{R}^{n}: w \in \boldsymbol{w}\right\}$. We call $C, \boldsymbol{w}$ and $\tilde{x}$ the characteristic matrix, box and vector of the parallelotope. The interval hull $\square \widehat{\boldsymbol{x}}$ is easily computed as $C \boldsymbol{w}+\tilde{x}$ using interval arithmetic. Parallelotopes are used in [9] in order to enclose and certify $b$-manifolds defined by a system $F(x)=0$, with $F: \mathbb{R}^{a+b} \rightarrow \mathbb{R}^{a}$. To do so, interval techniques are applied to characteristic boxes on an auxiliary system $G(w)=0$, with $G(w)=F(C w+\tilde{x})$, whose derivative is $G^{\prime}(w)=F^{\prime}(C w+\tilde{x}) C$. The last $b$ components of $w$ are identified to parameters: $w$ is split into $w=(u, v)$ with $u \in \mathbb{R}^{a}$ and $v \in \mathbb{R}^{b}$. The aim is to build parallelotopes $\widehat{\boldsymbol{x}}=$ $(C,(\boldsymbol{u}, \boldsymbol{v}), \tilde{x})$ that contain a solution for each parameters values in their domain, 

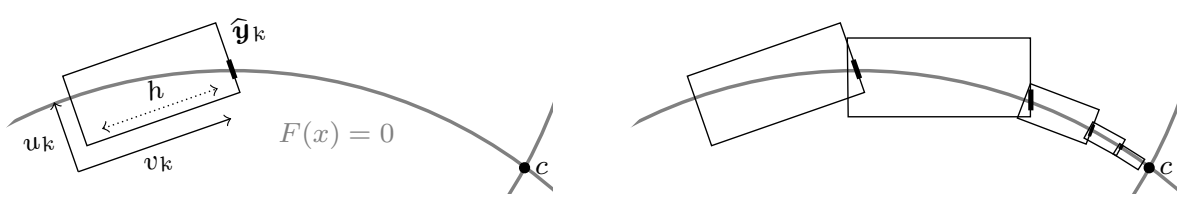

Fig. 1 Left: A parallelotope $\widehat{\boldsymbol{x}}_{k}=\left(C_{k},\left(\boldsymbol{u}_{k}, \boldsymbol{v}_{k}\right), \tilde{x}_{k}\right)$ constructed by ParCont. Left: Successive steps of ParCont. $c$ is a singular point of $F(x)=0$.

i.e. satisfying

$$
\forall v \in \boldsymbol{v}, \exists u \in \boldsymbol{u}, G(u, v)=0 .
$$

This property expresses that the manifold crosses the whole parallelotope along the parameter subspace. Proofs of this property can be obtained through a dedicated interval Newton method, see [9]. It also proves that the crossed manifold is continuously differentiable.

The Certified Continuation Method ParCont. In [22], the authors have proposed a certified and interval-based continuation method ParCont. The method constructs by continuation a paving with parallelotopes of a one-manifold, implicitly defined by a system $F(x)=0, F: \mathbb{R}^{n} \rightarrow \mathbb{R}^{n-1}$, hence avoiding the costly global search performed in [9]. The process is certified, i.e. the paving is proved to contain a unique continuous curve of solutions. ParCont can be seen as an intervalization of the arc-length continuation.

ParCont takes as input a system $F(x)=0$ implicitly defining a one-manifold, an approximate initial solution $x_{0}$ on the manifold and a search domain $\boldsymbol{x}^{\text {init }}$. It also considers a direction $d \in\{-1,1\}$ of continuation that allows performing the continuation in each direction from $x_{0}$. ParCont constructs and returns two sequences of parallelotopes $\left(\widehat{\boldsymbol{x}}_{1}, \ldots \widehat{\boldsymbol{x}}_{K}\right)$ and $\left(\widehat{\boldsymbol{y}}_{0}, \ldots, \widehat{\boldsymbol{y}}_{K}\right)$. Each parallelotope $\widehat{\boldsymbol{x}}_{k}$ is built such that it is proved being crossed by a unique solution curve from the input edge $\widehat{\boldsymbol{x}}_{k}^{i n}=\left(C_{k},\left(\boldsymbol{u}_{k}, \underline{v}_{k}\right), \tilde{x}_{k}\right)$ to the output edge $\widehat{\boldsymbol{x}}_{k}^{\text {out }}=\left(C_{k},\left(\boldsymbol{u}_{k}, \bar{v}_{k}\right), \tilde{x}_{k}\right)$. Each parallelotope $\widehat{\boldsymbol{y}}_{k}$ is a contraction of the output edge of $\widehat{\boldsymbol{x}}_{k}$, i.e. a tight enclosure of the single solution of the curve in $\widehat{\boldsymbol{x}}_{k}^{\text {out }}$. The exception being $\widehat{\boldsymbol{y}}_{0}$ which encloses the solution on the input edge of $\widehat{\boldsymbol{x}}_{1}$. Each iteration $k$ of ParCont builds a parallelotope $\widehat{\boldsymbol{x}}_{k}$ (and $\widehat{\boldsymbol{y}}_{k}$ ) in the direction of continuation and of length $h$ along the manifold, starting from $\widehat{\boldsymbol{y}}_{k-1}$. An iteration $k$ may fail, which induces a reduction of the length of the continuation step $h$, and the restart of the iteration $k$. Figure 1 displays several steps of ParCont. Singular solutions along the manifold cannot be certified, hence triggering a failure of the iteration. The step length is decreased accordingly, and therefore the method will conserve toward the singularity in case some are present on the manifold, as illustrated on Figure 1.

The algorithm stops once the tracked manifold provably 1) exits the search domain $\boldsymbol{x}^{\text {init }}, 2$ ) loops or 3 ) the step length $h$ becomes too short. Correctness, termination and asymptotic convergence of the method are proved in [22]. The complexity of one iteration of ParCont is $O\left(n^{3}\right)$. The total number of iterations depends on the length of the manifold within $\boldsymbol{x}^{\text {init }}$ and on the conditioning of $F$. 

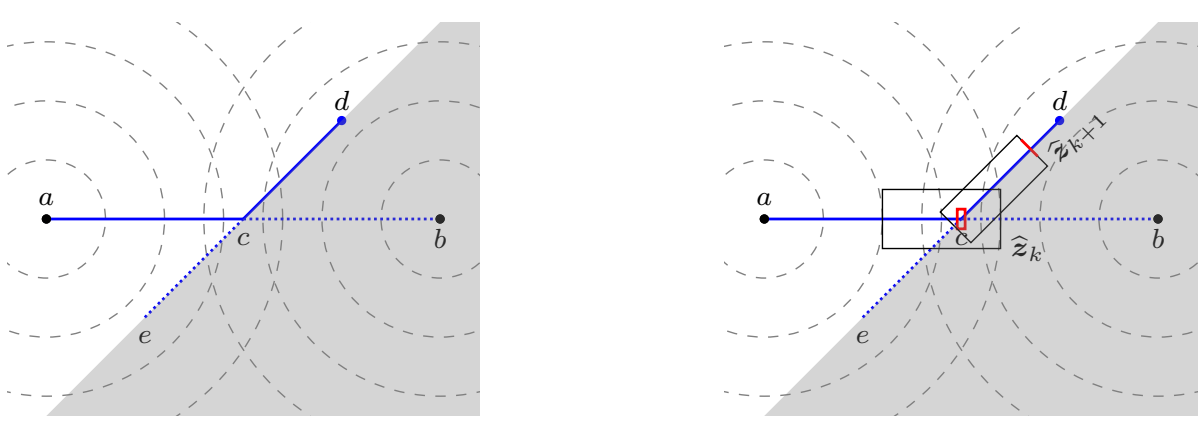

Fig. 2 Bi-objective problem of Example 1.

\subsection{Adapting ParCont to Bi-Objective Optimization}

Consider a NLBOO problem as (1) and its system $F$ of first order conditions as (5), involving variables $x \in \mathbb{R}^{n}$ and multipliers $\lambda \in \mathbb{R}_{+}^{2}, r \in \mathbb{R}_{+}^{p}$ and $s \in \mathbb{R}^{q}$. We denote by $z=(x, \lambda, r, s)$ the vector of these variables and multipliers and consider ParCont iterates on parallelotopes $\widehat{\boldsymbol{z}}_{k}$.

Some singularities in $F$ are triggered at changes of the active set of constraints, i.e. at solutions $z$ where there is a constraint $i$ with $g_{i}(x)=0$ and $r_{i}=0$. The equation $g_{i}(x) r_{i}=0$ actually consists of the product of two zero quantities. Hence, two paths are possible, one where $g_{i}(x) \neq 0$ and $r_{i}=0$ (only the part $g_{i}(x) \leq 0$ is feasible), the other where $g_{i}(x)=0$ and $r_{i} \neq 0$ (only the part $r_{i} \geq 0$ is feasible).

Example 1 Consider the bi-objective problem with $f_{1}(x)=\left(x_{1}+1\right)^{2}+x_{2}^{2}, f_{2}(x)=$ $\left(x_{1}-1\right)^{2}+x_{2}^{2}$ and inequality constraint $g(x)=x_{1}-x_{2} \leq 0$. Variables of $F$ are here $z=\left(x_{1}, x_{2}, \lambda_{1}, \lambda_{2}, r\right)$. This problem is illustrated in the left hand side graphic of Figure 2 in the plane $\left(x_{1}, x_{2}\right)$. The solution $c=(0,0, \sqrt{2} / 2, \sqrt{2} / 2,0)$ is singular for $F$. The curve of solutions bifurcates at this point in two paths: The path from $a=$ $(-1,0,1,0,0)$ to $b=(1,0,0,1,0)$ satisfies $r=0$ but is infeasible from $c$ to $b(g(x)>$ $0)$; the path from $d=(0.5,0.5,0, \sqrt{2} / 2, \sqrt{2} / 2)$ to $e=(-0.5,-0.5, \sqrt{2} / 2,0,-\sqrt{2} / 2)$ satisfies $g(x)=0$ but is infeasible from $c$ to $e(r<0)$.

Given a set $\mathcal{A}$ of active constraints, it is known (see [34]) that applying a continuation on the full system of first order conditions (5) is equivalent to applying it on a reduced system involving only the active constraints:

$$
F_{\mathcal{A}}(x, \lambda, r, s)=\left(\begin{array}{l}
\nabla f(x) \lambda+\nabla g_{\mathcal{A}}(x) r_{\mathcal{A}}+\nabla h(x) s \\
(\forall i \in \mathcal{A}) g_{i}(x) \\
(\forall i=1, \ldots, q) h_{i}(x) \\
\lambda^{T} \lambda+r^{T} r+s^{T} s-1
\end{array}\right)=0,
$$

with $x \in \mathcal{X}, r \geq 0$ and $\lambda \geq 0$. Note that $r_{i}=0$ for all $i \notin \mathcal{A}$. As ParCont cannot handle singularities, it is preferable to apply it on (12) than on (5), but it requires managing correctly the active set.

Let $\mathcal{A}_{k}$ be the set of active constraints at iteration $k$ of ParCont, and $\widehat{\boldsymbol{z}}_{k}=$ $\left(C_{k},\left(\boldsymbol{u}_{k}, \boldsymbol{v}_{k}\right), \tilde{z}_{k}\right)$ a parallelotope certified to contain solutions of (12), i.e. condition (11) holds in $\widehat{\boldsymbol{z}}_{k}$. In order to maintain a correct active set for the next 
iteration of ParCont, it is required to determine changes in $\mathcal{A}_{k}$ that occur on the solution curve contained in $\widehat{\boldsymbol{z}}_{k}$. To this end, we introduce the following Constraint Satisfaction Problems (CSP) for each constraint $g_{i}$ :

$$
\left[\begin{array}{l}
z=C_{k}(u, v)+\tilde{z}_{k}, F_{\mathcal{A}_{k}}(z)=0, \gamma_{i}(z)=0 \\
u \in \boldsymbol{u}_{k}, v \in \boldsymbol{v}_{k}
\end{array}\right]
$$

whose variables $u$ and $v$ take their values in $\boldsymbol{u}_{k}$ and $\boldsymbol{v}_{k}$ respectively, i.e. the characteristic box of $\widehat{\boldsymbol{z}}_{k}$. The virtual variables $z$, and the first constraint, express the transition from the parallelotope basis. The second constraint enforces (12). The third constraint deals with the activation/disactivation of inequality $g_{i}$ : The function $\gamma_{i}(z)$ is defined as $g_{i}(x)$ if $i \notin \mathcal{A}_{k}$, or as $r_{i}$ if $i \in \mathcal{A}_{k}$. Since (11) holds for (12) in $\widehat{\boldsymbol{z}}_{k}$, there is an unique solution to the equations $F_{\mathcal{A}_{k}}(z)=0$ for each $v \in \boldsymbol{v}_{k}$. Hence, solving (13) can be done by performing an unidimensional search in the domain $\boldsymbol{v}_{k}$ of variable $v$, whose complexity is generally low. In addition, since only the first change of activity is of interest, a single solution is computed, the one closest to $\underline{v}_{k}$.

Changes in the active set must be certified for ParCont to remain rigorous. To this end, we propose to solve (13) with an interval-based branch and prune method, adapted to the specificities of this problem (i.e. it branches only on the domain $\boldsymbol{v}_{k}$ of the variable $v$, and uses only the interval Newton). For each CSP (13), the method returns either no solution, thus proving no change of activity occurs in $\widehat{\boldsymbol{z}}_{k}$ for this constraint; or one certified solution box $\left(\boldsymbol{u}_{k}^{i}, \boldsymbol{v}_{k}^{i}\right)$ identifying a verified change; or one non-certified solution box if the computational precision is insufficient.

At each iteration of ParCont, once a parallelotope $\widehat{\boldsymbol{z}}_{k}$ has been certified, a CSP (13) is solved for each constraint $g_{i}$. Only the first change among all the returned (certified or not) boxes $\left(\boldsymbol{u}_{k}^{i}, \boldsymbol{v}_{k}^{i}\right)$ is considered. If at least two computed boxes are candidates (the solutions boxes are overlapping), or if the first box is non-certified, we fail to certify a change of the active set, a failure of the iteration $k$ of ParCont is triggered and the step size is reduced. Therefore, we cannot decide a situation where two activation changes occur at the same place ${ }^{1}$. Otherwise, a change has been certified. The corresponding box $\left(\boldsymbol{u}_{k}^{i}, \boldsymbol{v}_{k}^{i}\right)$ is used to build $\widehat{\boldsymbol{y}}_{k}$, the output edge parallelotope of $\widehat{\boldsymbol{z}}_{k}$, and the active set $\mathcal{A}_{k+1}$ for the next iteration can be constructed accordingly. Note that if the activation status of the constraint $g_{i}$ has changed on the previous step of ParCont then the first solution is the reverse change of activity of this constraint, which must be avoided. In this case, the second change of activity of $g_{i}$ has to be considered instead of the first. The two parallelotopes built following this procedure are illustrated on the right hand side graphic of Figure 2.

This strategy for maintaining the correct active set of constraint has been incorporated in ParCont. The stopping criterion of ParCont based on the search domain only considers the domains of $\lambda_{1}$ and $\lambda_{2}$ (i.e. $\lambda_{1}, \lambda_{2} \geq 0$ ), the non-negativeness of the multipliers $r_{i}$ being maintained by the active set strategy. Note that the proposed adaptation only deals with singularities of (5) induced by a change of the active set, but other singularities, such as the loss of constraint qualification, cannot be handled.

1 Note that such coincident changes of activation are unstable, since an arbitrarily small perturbation of the constraint can remove it. Therefore, they cannot be handled numerically with certification. 


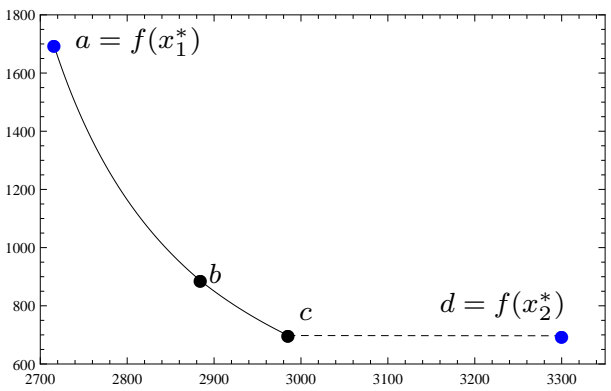

Fig. 3 Captured Pareto-optimal curve of (14) in the objective space.

\subsection{Illustrative Experiment}

ParCont and its adaptation to NLBOO have been implemented in $\mathrm{C}++$ using the RealPaver [11] API, implementing routines such as interval Newton methods and many other constraint solving techniques, and using Gaol [10] for interval arithmetic. This experiment has been run on a computer under Linux Ubuntu version 11.10, with processor Intel i5-2400 3.10GHz and 4Gb of RAM. Parameters of ParCont are tuned as prescribed in [22].

We consider the bi-objective problem from [44] for the design of a speech reducer:

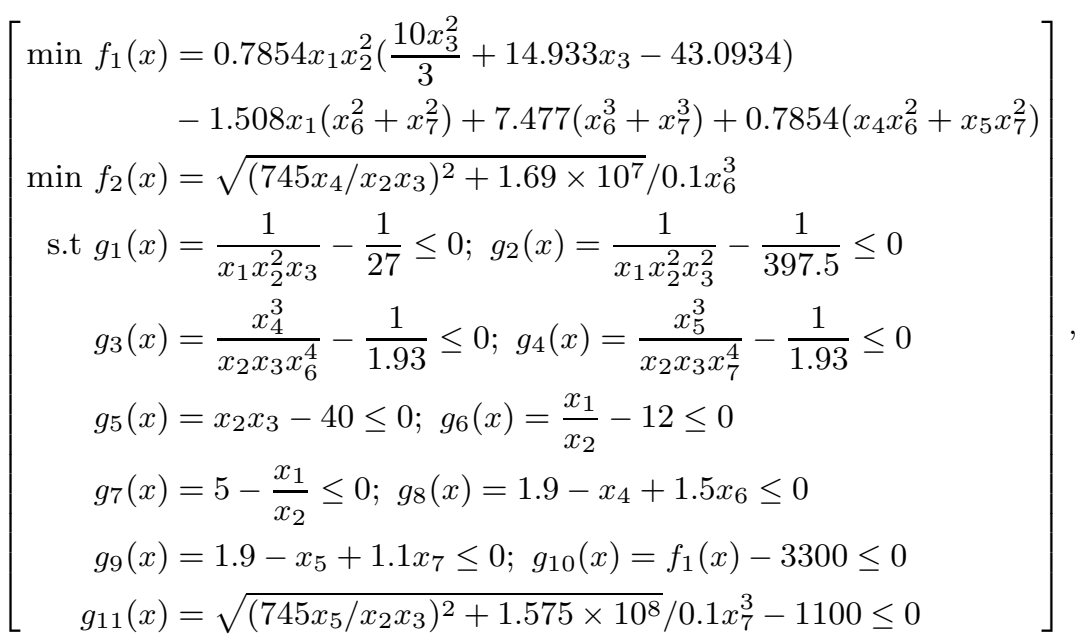

with $2.6 \leq x_{1} \leq 3.6,0.7 \leq x_{2} \leq 0.8,17 \leq x_{3} \leq 28,7.3 \leq x_{4}, x_{5} \leq 8.3,2.9 \leq x_{6} \leq 3.9$ and $5 \leq x_{7} \leq 5.5$. Note that the variables domains are considered as inequality constraints. They will be denoted $\underline{g}_{x_{i}}$ and $\bar{g}_{x_{i}}$ for respectively the lower and upper bound constraint of $x_{i}$. Last, we consider a relaxed problem: The variable $x_{3}$, originally an integer, is consider as a real variable. We apply ParCont at two different starting points: The minimum of $f_{1}$ and the minimum of $f_{2}$. The obtained Pareto-front is shown in Figure 3. ParCont has taken 0.25 seconds and produced 48 parallelotopes to obtain these results.

The points $a, b, c$ and $d$ correspond to solutions on which a change of $\mathcal{A}$ occurs. The first application of ParCont computes an enclosure from $a$ to $c$, and the second 
from $d$ to $c$ (these two curves are actually connected by solution with negative $\lambda$ multipliers). The obtained solutions show that the constraints $g_{7}, g_{9}, \underline{g}_{x_{2}}$ and $\underline{g}_{x_{7}}$ are always active. Constraints reported in Table 1 change activity during the process, the others are inactive along the Pareto front.

Table 1 Change of $\mathcal{A}$ : + means activated, - means disactivated

\begin{tabular}{l|ccccc|ccc} 
Constraint & $a \rightarrow a$ & $a \rightarrow b$ & $b \rightarrow b$ & $b \rightarrow c$ & $c \rightarrow c$ & $c \leftarrow c$ & $c \leftarrow d$ & $d \leftarrow d$ \\
\hline$g_{8}$ & - & - & - & + & + & + & + & + \\
$g$ & + & + & + & - & - & - & - & - \\
$g_{x_{4}}$ & + & - & - & - & - & - & - & - \\
$\bar{g}_{x_{6}}$ & - & - & - & - & + & + & + & + \\
$g_{x_{3}}$ & + & + & + & + & + & + & - & - \\
$g_{10}$ & - & - & - & - & - & - & - & +
\end{tabular}

Consider first the path from $a$ to $c$. Table 1 shows that 7 constraints, for 7 variables $x$, are active at its start $a$. Hence, the active set of constraints form a square system of equations uniquely defining the values of $x$. In such situation, the Pareto manifold remains constant in $x$, but moves in the multipliers space $\left(\lambda, r_{\mathcal{A}}\right)$. The disactivation of $\underline{g}_{x_{6}}$ is finally observed, allowing the continuation to exit $a$. On the path $a \rightarrow b, 6$ constraints are active, hence the continuation tracks other solutions in the variable space, and in the objective space. When reaching $b, g_{8}$ activates. Again, 7 constraints are active such that a path $b \rightarrow b$ is followed in the multiplier space until the detection of the disactivation of $\underline{g}_{x_{4}}$. The path $b \rightarrow c$ has 6 active constraints, and reaches $c$ by activating the constraint $\bar{g}_{x_{6}}$.

Consider now the path from $d$ to $c$. As for the other starting solution, 7 constraints are active at $d$. A path $d \rightarrow d$ in the multipliers space is followed until the disactivation of $g_{10}$. Then, the continuation tracks solutions from $d \rightarrow c$, and reaches $c$ by activating the constraint $\underline{g}_{x_{3}}$. The point $c$ shares the same $x$ values in both paths. However, those paths are connected by solutions having negative objective multipliers $(\lambda<0)$. Authorizing negative $\lambda$ would allow connecting those curves, but it may result in producing non Pareto-optimal solutions elsewhere.

\section{Conclusion}

We have presented in this paper an overview of continuation techniques for NLBOO problems: local techniques that follow curves of (locally) Pareto-optimal solutions. Techniques based on the framework in [15], i.e. applying a continuation process on the system of optimality conditions, show a better behavior on nonconvex problems than other approaches, like methods based on the local solving of scalarized problems. However the former approaches generally do not handle inequality constraints.

To overcome this limitation, we have introduced a certified continuation method for NLBOO, based on interval analysis, that handles inequality constraints through an active set management process. It certifies changes of constraints activity provided that these changes occur one at a time. Applied to a complex constrained problem, the approach shows its ability to track the Pareto-optimal curves with 
guarantee of feasibility and optimality with respect to the first-order conditions. Such certificates of feasibility are helpful in global solvers, e.g. population based algorithms or branch and bound, as they can be used to accurately and rigorously construct bounds.

\section{References}

1. E. Allgower and K. Georg. Numerical continuation methods, volume 33. Springer, 1990.

2. C. Beltrán and A. Leykin. Certified numerical homotopy tracking. Experimental Mathematics, 21(1):69-83, 2012.

3. W.-J. Beyn, C. Effenberger, and D. Kressner. Continuation of eigenvalues and invariant pairs for parameterized nonlinear eigenvalue problems. Numerische Mathematik, 119:489516, 2011.

4. J.-D. Boissonnat and A. Ghosh. Triangulating smooth submanifolds with light scaffolding. Math. in Computer Science, 4:431-461, 2010.

5. I. Das and J. Dennis. Normal-Boundary Intersection: A New Method for Generating the Pareto Surface in Nonlinear Multicriteria Optimization Problems. SIAM Journal on Optimization, 8(3):631-657, 1998.

6. M. Ehrgott. Multicriteria Optimization (2. ed.). Springer, 2005.

7. T. Erfani and S. Utyuzhnikov. Directed search domain: a method for even generation of the pareto frontier in multiobjective optimization. Engineering Optimization, 43(5):467-484, 2011.

8. D. Faudot and D. Michelucci. A new robust algorithm to trace curves. Reliable Computing, 13(4):309-324, 2007.

9. A. Goldsztejn and L. Granvilliers. A new framework for sharp and efficient resolution of NCSP with manifolds of solutions. Constraints, 15(2):190-212, 2010

10. F. Goualard. GAOL 3.1.1: Not Just Another Interval Arithmetic Library. LINA, 4.0 edition, 2006

11. L. Granvilliers and F. Benhamou. Algorithm 852: RealPaver: an interval solver using constraint satisfaction techniques. ACM Trans. Mathematical Software, 32(1):138-156, 2006.

12. K. Harada, J. Sakuma, and S. Kobayashi. Local search for multiobjective function optimization: pareto descent method. In GECCO, pages 659-666. ACM, 2006.

13. K. Harada, J. Sakuma, S. Kobayashi, and I. Ono. Uniform sampling of local pareto-optimal solution curves by pareto path following and its applications in multi-objective GA. In GECCO, pages 813-820. ACM, 2007.

14. K. Harada, J. Sakuma, I. Ono, and S. Kobayashi. Constraint-handling method for multiobjective function optimization: Pareto descent repair operator. In Evolutionary MultiCriterion Optimization, volume 4403 of LNCS, pages 156-170. Springer, 2007.

15. C. Hillermeier. Generalized homotopy approach to multiobjective optimization. J. Optimization Theory and Applications, 110(3):557-583, 2001.

16. B. Kearfott and Z. Xing. An interval step control for continuation methods. SIAM J. Numerical Analysis, 31(3):pp. 892-914, 1994.

17. A. Lara, G. Sanchez, C. Coello Coello, and O. Schütze. HCS: A new local search strategy for memetic multiobjective evolutionary algorithms. IEEE Trans. Evolutionary Computation, 14(1):112-132, 2010.

18. F. Logist and J. Van Impe. Novel insights for multi-objective optimisation in engineering using Normal Boundary Intersection and (Enhanced) Normalised Normal Constraint. Structural and Multidisciplinary Optimization, 45(3):417-431, 2012.

19. A. Lovison. Singular continuation: Generating piecewise linear approximations to pareto sets via global analysis. SIAM J. Optimization, 21(2):463-490, 2011.

20. A. Lovison. Global search perspectives for multiobjective optimization. J. Global Optimization, pages 1-14, 2012.

21. B. Lundberg and A. Poore. Numerical continuation and singularity detection methods for parametric nonlinear programming. SIAM J. Optimization, 3(1):134-154, 1993.

22. B. Martin, A. Goldsztejn, L. Granvilliers, and C. Jermann. Certified parallelotope continuation for one-manifolds. SIAM Journal on Numerical Analysis, 2013. (to appear).

23. Jean-Pierre Merlet. Parallel robots. Kluwer, 2000. 
24. A. Messac, A. Ismail-Yahaya, and C. Mattson. The normalized normal constraint method for generating the pareto frontier. Structural and Multidisciplinary Optimization, 25(2):86-98, 2003.

25. A. Messac and C. Mattson. Generating well-distributed sets of pareto points for engineering design using Physical Programming. Optimization and Engineering, 3(4):431-450, 2002.

26. A. Messac and C. Mattson. Normal constraint method with guarantee of even representation of complete pareto frontier. AIA A Journal, 42:2101-2111, 2004.

27. K. Miettinen. Nonlinear Multiobjective Optimization, volume 12 of Int. Series in Operations Research and Management Science. Kluwer, 1999.

28. R. Motta, S. Afonso, and P. Lyra. A modified NBI and NC method for the solution of N-multiobjective optimization problems. Structural and Multidisciplinary Optimization, 46(2):239-259, 2012.

29. A. Neumaier. Interval Methods for Systems of Equations. Cambridge University Press, 1991.

30. V. Pereyra. Fast computation of equispaced pareto manifolds and pareto fronts for multiobjective optimization problems. Mathematics and Computers in Simulation, 79(6):19351947, 2009.

31. J.-M. Porta, L. Jaillet, and O. Bohigas. Randomized path planning on manifolds based on higher-dimensional continuation. I. J. Robotics Research, 31(2):201-215, 2012.

32. A. Potschka, F. Logist, J. Van Impe, and H. Bock. Tracing the Pareto frontier in biobjective optimization problems by ODE techniques. Numerical Algorithms, 57(2):217$233,2011$.

33. J. Rakowska, R. Haftka, and L. Watson. An active set algorithm for tracing parametrized optima. Structural optimization, 3(1):29-44, 1991.

34. J. Rakowska, R. Haftka, and L. Watson. Multi-objective control-structure optimization via homotopy methods. SIAM J. Optimization, 3(3):654-667, 1993.

35. J. Rao and P. Papalambros. A non-linear programming continuation strategy for one parameter design optimization problems. In ASME Design Automation Conference, pages 77-89, 1989.

36. E. Rigoni and S. Poles. NBI and MOGA-II, two complementary algorithms for multiobjective optimizations. In Practical Approaches to Multi-Objective Optimization, number 04461 in Dagstuhl Seminar, 2005.

37. M. Ringkamp, S. Ober-Blbaum, M. Dellnitz, and O. Schütze. Handling high-dimensional problems with multi-objective continuation methods via successive approximation of the tangent space. Engineering Optimization, 44(9):1117-1146, 2012.

38. J. Sanchis, M. Martínez, X. Blasco, and J. Salcedo. A new perspective on multiobjective optimization by enhanced normalized normal constraint method. Structural and Multidisciplinary Optimization, 36(5):537-546, 2008.

39. O. Schütze, C. Coello Coello, S. Mostaghim, E. Talbi, and M. Dellnitz. Hybridizing evolutionary strategies with continuation methods for solving multi-objective problems. Engineering Optimization, 40:383-402, 2008.

40. O. Schütze, A. Dell'Aere, and M. Dellnitz. On continuation methods for the numerical treatment of multi-objective optimization problems. In J. Branke, K. Deb, K. Miettinen, and R. Steuer, editors, Practical Approaches to Multi-Objective Optimization, number 04461 in Dagstuhl Seminar, 2005.

41. O. Schütze, A. Lara, and C. Coello Coello. Evolutionary continuation methods for optimization problems. In GECCO, pages 651-658. ACM, 2009.

42. S. Smale. Newton's method estimates from data at one point. In The Merging of Disciplines in Pure, Applied and Computational Mathematics, pages 185-196. Springer, 1986.

43. S. Utyuzhnikov, P. Fantini, and M. Guenov. A method for generating a well-distributed pareto set in nonlinear multiobjective optimization. J. Computational and Applied Mathematics, 223(2):820 - 841, 2009.

44. Z. Zhang. Immune optimization algorithm for constrained nonlinear multiobjective optimization problems. Applied Soft Computing, 7(3):840 - 857, 2007. 